\title{
P2Y12 platelet inhibition in clinical practice
}

\author{
Peter Damman • Pier Woudstra • Wichert J. Kuijt • \\ Robbert J. de Winter $\cdot$ Stefan K. James
}

Published online: 20 December 2011

(C) The Author(s) 2011. This article is published with open access at Springerlink.com

\begin{abstract}
Platelet adhesion, activation and aggregation play a pivotal role in atherothrombosis. Intracoronary atherothrombosis is the most common cause of the development of acute coronary syndrome (ACS), and plays a central role in complications occurring around percutaneous coronary intervention (PCI) including recurrent ACS, procedure-related myocardial infarction or stent thrombosis. Inhibition of platelet aggregation by medical treatment impairs formation and progression of thrombotic processes and is therefore of great importance in the prevention of complications after an ACS or around PCI. An essential part in the platelet activation process is the interaction of adenosine diphosphate (ADP) with the platelet P2Y12 receptor. The $\mathrm{P} 2 \mathrm{Y} 12$ receptor is the predominant receptor involved in the ADP-stimulated activation of the glycoprotein IIb/IIIa receptor. Activation of the glycoprotein IIb/ IIIa receptor results in enhanced platelet degranulation and thromboxane production, and prolonged platelet aggregation. The objectives of this review are to discuss the pharmacological limitations of the P2Y12 inhibitor clopidogrel, and describe the novel alternative P2Y12 inhibitors prasugrel and ticagrelor and the clinical implications of the introduction of these new medicines.
\end{abstract}

P. Damman · P. Woudstra - W. J. Kuijt · R. J. de Winter Department of Cardiology, Academic Medical Center, University of Amsterdam, Amsterdam, The Netherlands

P. Damman · S. K. James ( $\square)$

Uppsala Clinical Research Center, Dag Hammarskjölds väg 50A, MTC-huset, Science Park, 6363, 75185 Uppsala, Sweden e-mail: Stefan.James@ucr.uu.se

S. K. James

Department of Medical Sciences, Uppsala University,

Uppsala, Sweden
Keywords P2Y12 inhibitors · Ticagrelor · Prasugrel · Clopidogrel

\section{Introduction}

Platelet adhesion, activation and aggregation play a pivotal role in atherothrombosis. Intracoronary atherothrombosis is the most common cause of the development of acute coronary syndrome (ACS), and plays a central role in complications occurring around percutaneous coronary intervention (PCI) including recurrent ACS, procedurerelated myocardial infarction (MI) or stent thrombosis [1]. Inhibition of platelet aggregation by medical treatment impairs formation and progression of thrombotic processes and is therefore of great importance in the prevention of complications after an ACS or around PCI [2,3]. Platelet inhibitors include thromboxane inhibitors (aspirin); adenosine diphosphate (ADP) receptor antagonists (or P2Y12 inhibitors) such as the thienopyridines (clopidogrel and prasugrel) and the nonthienopyridines (elinogrel, ticagrelor and cangrelor); the glycoprotein IIb/IIIa inhibitors; and medication working through other pathways and include dipyridamole, cilostazol, protease-activated receptor antagonists and the platelet adhesion antagonists [4].

The platelet inhibitor of choice, the optimal time of initiation and the duration of treatment depend on the indication for therapy and patient characteristics. In recent years, bleeding has been identified as an important risk factor for adverse outcomes and has led to a renewed emphasis on individual bleeding risk in choosing appropriate therapy [5]. Current European treatment guidelines recommend dual antiplatelet therapy with aspirin and clopidogrel for patients with ACS and patients who undergo PCI with stent placement [2]. The efficacy of this 
combination in patients with ST-segment elevation myocardial infarction (STEMI), non-ST-segment elevation myocardial infarction (NSTEMI) or unstable angina has been shown in multiple large clinical trials [6-8]. Despite the efficacy of the aforementioned combination, there are important pharmacological limitations associated with clopidogrel use. Newly developed P2Y12 inhibitors such as prasugrel, ticagrelor and cangrelor, are more potent and have a faster onset of action than clopidogrel. The objectives of this review are to discuss the limitations of clopidogrel, and describe alternative P2Y12 inhibitors and the clinical implications of the introduction of these new medicines.

\section{Pharmacology of P2Y12 inhibitors}

An essential part in the platelet activation process is the interaction of ADP with the platelet $\mathrm{P} 2 \mathrm{Y} 12$ receptor [9]. The $\mathrm{P} 2 \mathrm{Y} 12$ receptor is the predominant receptor involved in the ADP-stimulated activation of the glycoprotein IIb/ IIIa receptor [10]. Activation of the glycoprotein IIb/IIIa receptor results in enhanced platelet degranulation and thromboxane production, and prolonged platelet aggregation [11]. Thienopyridines inhibit the platelet activation and aggregation by antagonizing the platelet $\mathrm{P} 2 \mathrm{Y} 12$ receptor. This prevents the binding of ADP to the receptor which attenuates platelet aggregation and reaction of platelets to stimuli of thrombus aggregation such as thrombin [4].

\section{Pharmacological limitations of clopidogrel}

Despite proven clinical efficacy of clopidogrel in patients with an ACS or after PCI, either as monotherapy or in combination with aspirin, pharmacological limitations of clopidogrel prevent this medication from always being fully effective [12]. Clopidogrel, a prodrug, requires a 2-step hepatic cytochrome P450 (CYP) metabolic activation to produce the active metabolite that inhibits the platelet P2Y12 receptor [13]. Before intestinal absorption, $85 \%$ of the pro-drug is hydrolyzed by esterases to an inactive carboxylic acid derivate. Because of these pharmacodynamic characteristics of clopidogrel, several hours pass between ingestion and reaching therapeutic levels. This results in suboptimal platelet aggregation inhibition during acute PCI for ACS and a higher risk for acute stent thrombosis. Moreover, the longer period up to therapeutic levels may raise the bleeding risk during acute coronary artery bypass grafting (CABG) if necessary based on coronary anatomy. Second, there is a substantial variability in clopidogrel response between patients. Accumulating evidence shows that a suboptimal response to clopidogrel is associated with worse clinical outcomes such as coronary ischemia or stent thrombosis [14-17]. This suboptimal therapeutic response is a consequence of the variation in the CYP gene [18]. This gene codes for the CYP-450 enzymes involved in the biotransformation of the prodrug clopidopgrel to the active metabolite. Particularly polymorphisms in the CYP2C19 allele are associated with a reduced activity of clopidogrel. Three large studies have shown that clopidogrel users who are carrier of the loss-offunction CYP2C19 allele endure more ischemic events compared with patients without this mutation, with the genetic substudy of the PLATO (Study of Platelet Inhibition and Patient Outcomes) trial showing that these were mainly early events (within 30 days) [19-21]. Treatment strategies tailored to the heterogeneity in clopidogrel response were investigated in the GRAVITAS (Gauging Responsiveness with A VerifyNow Assay-Impact on Thrombosis and Safety) trial [22]. This trial analyzed whether tailored platelet aggregation inhibition on the basis of platelet function testing using a point-of-care assay (VerifyNow, Accumetrics Inc, San Diego, CA, USA) improved cardiovascular outcomes after drug-eluting stent placement during urgent or elective PCI. However, in patients with a high residual platelet activity, a higher clopidogrel dosing (loading dosis $600 \mathrm{mg}$ post-PCI, followed by $150 \mathrm{mg}$ daily) did not reduce the incidence of ischemic outcomes compared with a lower clopidogrel dosing (loading doses $150 \mathrm{mg}$ post-PCI, followed by $75 \mathrm{mg}$ daily). In order to overcome the described limitations of clopidogrel, alternative antiplatelet treatments have been developed.

\section{Pharmacology of prasugrel}

Prasugrel is a third generation thienopyridine. Rapidly after ingestion, prasugrel is hydrolyzed in the gastro-intestinal system into an intermediary metabolite. This intermediary metabolite is hepatically activated in a single-step and forms an active metabolite that binds to the P2Y12 receptor on the platelet. This irreversible bond with the receptor inhibits activation and aggregation of the platelet [18]. The peak concentration of the active metabolite of prasugrel is reached after $30 \mathrm{~min}$, and the final concentration is linearly dependent on the prasugrel dose which varies between 5 and $60 \mathrm{mg}$. If not bound to the receptor, active metabolites have a half life of approximately $7 \mathrm{~h}$ [18]. A maximum of 60-70\% platelet inhibition is usually achieved within 2-4 h [11]. There are important differences in the metabolic process between prasugrel and clopidogrel. First, with the initial hydrolization of clopidogrel, a substantial fraction is inactivated. Second, the activation of clopidogrel involves two CYP dependent steps, in contrast to a single CYP-dependent step with prasugrel [23]. This results in a 
more rapid and consistent activation of prasugrel, with more receptor blocking active metabolite [24]. Third, genetic CYP variants do not have a significant influence on the active metabolites of prasugrel, subsequent platelet aggregation and clinical outcomes [25].

As a consequence, more effective platelet aggregation is achieved with prasugrel compared with clopidogrel both after the loading dose and with maintenance dose, as shown by research in patients with stable coronary disease [25] and elective PCI [26].

Pharmacology of the direct-acting P2Y12 inhibitors ticagrelor and cangrelor

Ticagrelor is a compound that directly and reversibly binds to and inhibits the P2Y12 receptor at a site distinct from the ADP binding site [11]. The compound is orally active without the requirement of metabolic activation [27]. It undergoes enzymatic degradation to at least one active metabolite which is approximately as potent as its parent compound [27]. The maximum plasma concentration and maximum platelet inhibition is reached $1-3 \mathrm{~h}$ after treatment, and the plasma half-life is 6-13 h [11]. In patients with ACS, ticagrelor exhibited greater inhibition of platelet aggregation than a standard regimen of clopidogrel [28]. Moreover, CYP2C19 and ABCB1 genotypes, known to influence the effects of clopidogrel, did not influence the effect on ischemic outcomes in ACS patients [20].

Much like to ticagrelor, the intravenous agent cangrelor directly and reversibly antagonizes ADP binding to the P2Y12 receptor. Cangrelor rapidly reaches steady state plasma levels and platelet aggregation inhibition within 30 min of onset of infusion without the need for a bolus dose, and the plasma half-life is short, being approximately less than 9 min [29]. Maximal platelet inhibition is achieved within $15 \mathrm{~min}$. In patients with ischemic heart disease a substantially greater P2Y12 receptor blockade was achieved with cangrelor compared with clopidogrel [30].

\section{Clinical trials}

\section{Prasugrel}

The more effective platelet inhibition with the new P2Y12 inhibitors potentially results in a reduction of ischemic events and, the downside, more bleeding events. The safety and antiplatelet effects of prasugrel were investigated in two phase II studies, the JUMBO-TIMI 26 [31] and the PRINCIPLE-TIMI 44 [26]. The JUMBO-TIMI (Joint Utilization of Medications to Block Platelets OptimallyThrombolysis in Myocardial Infarction) 26 was a dose-ranging safety trial comparing different prasugrel doses with clopidogrel (loading dose $300 \mathrm{mg}$, maintenance dose $75 \mathrm{mg}$ ) in patients undergoing PCI [31]. At 30 days after PCI, no statistical difference was observed in nonCABG-related (TIMI major plus minor) bleeding events when comparing prasugrel with clopidogrel. However, bleeding events were numerically higher with prasugrel use. Access site bleeding occurred most frequently. Importantly, because of evidence available at the time of enrolment in JUMBO-TIMI 26, there was an increased use of higher-than-approved doses of clopidogrel (loading dose $600 \mathrm{mg}$ ) in clinical practice. In the second phase II study involving prasugrel, the PRINCIPLE-TIMI (Prasugrel in Comparison to Clopidogrel for Inhibition of Platelet Activation and Aggregation-TIMI) 44, a $60 \mathrm{mg}$ prasugrel dose was compared with a $600 \mathrm{mg}$ loading dose of clopidogrel [26]. Among patients planned for PCI, loading with $60 \mathrm{mg}$ prasugrel resulted in greater platelet inhibition than a $600 \mathrm{mg}$ clopidogrel loading dose. Daily maintenance therapy with prasugrel $10 \mathrm{mg}$ resulted in a greater antiplatelet effect than $150 \mathrm{mg}$ daily clopidogrel.

As in the previous investigation, numerically higher bleeding was observed with prasugrel, although this difference did not reach statistical significance. The overall positive results from the JUMBO-TIMI 26 trial resulted in a large phase III efficacy and safety trial, the TRITONTIMI (Trial to Assess Improvement in Therapeutic Outcomes by Optimizing Platelet Inhibition with Prasugrel-TIMI) 38 [32]. In this trial, 13,608 patients with moderate and high-risk ACS were randomized to prasugrel $(60 \mathrm{mg}$ loading dose and $10 \mathrm{mg}$ daily maintenance dose) or clopidogrel (300 mg loading dose and $75 \mathrm{mg}$ daily maintenance dose) for 6-15 months (median 14.5 months). Randomization took place after coronary angiography and before PCI. The main efficacy endpoint was cardiovascular death, nonfatal MI, or nonfatal stroke. Major bleeding (definition: TIMI major bleeding not related to $\mathrm{CABG}$ ), was included as the key safety endpoint. The use of prasugrel was associated with a significant reduction of the main efficacy endpoint, with an event rate of $12.1 \%$ in the clopidogrel group versus $9.9 \%$ in the prasugrel group $(P<0.001)$. This was mainly driven by a reduction in MI and stent thrombosis, no difference was observed in mortality. However, the reduction in ischemic endpoints with prasugrel was accompanied by a higher incidence of TIMI defined major bleeding, occurring in $1.8 \%$ of the patients in the clopidogrel group versus $2.4 \%$ in the prasugrel group. This translates into a risk of three extra major bleeds per 1,000 patients with prasugrel use, keeping in mind that patients at high risk for bleeding were excluded. As a consequence the bleeding risk with prasugrel could be potentially higher in clinical practice, although the same is true for the benefits [33]. This balance 
has received increasing attention as many of the established risk factors for ischemic events are also suggestive of higher bleeding risk [34]. Especially noteworthy in this regard are the higher incidence of fatal and CABG-related bleeding observed with prasugrel. In an exploratory analysis, three subgroups of interest were identified that had less clinical efficacy and greater absolute levels of bleeding than the overall cohort, resulting in less net clinical benefit or in clinical harm. These included patients with a history of stroke or transient ischemic attack, patients aged 75 years and older, and patients with a body weight of less than $60 \mathrm{~kg}$. Subanalyses from the TRITON-TIMI 38 are summarized in Table 1.

\section{Ticagrelor}

The safety, tolerability and efficacy of ticagrelor were investigated in the DISPERSE-2 (Dose confirmation study assessing anti-platelet effects of AZD6140 vs. clopidogRel in non-ST-segment Elevation myocardial infarction) phase II trial [35]. In this trial patients with a NSTE-ACS were randomized to receive ticagrelor 90 or $180 \mathrm{mg}$ twice a day, or clopidogrel $75 \mathrm{mg}$ once a day for up to 12 weeks. At 4-week follow-up, no difference was observed in major bleeding although an increase in minor bleeding was observed at the higher ticagrelor dose. On the other side, encouraging results were observed on the secondary end point of MI. Both doses of ticagrelor achieved a greater mean inhibition of platelet aggregation than clopidogrel in the ACS patients [28]. Ticagrelor was compared with clopidogrel in 18,624 patients with ACS in the multicenter randomized PLATO (Study of Platelet Inhibition and Patient Outcomes) [36]. Patients on maintenance treatment or who had received loading doses of clopidogrel were accepted. After randomization, the patients received ticagrelor (180 mg loading dose, $90 \mathrm{mg}$ twice daily thereafter) or clopidogrel (300-600 mg loading dose, $75 \mathrm{mg}$ daily thereafter). Patient randomization took place as early as possible after the index event. The main outcome at 12-month follow-up was the composite of cardiovascular death, MI or stroke which occurred in $9.8 \%$ of patients receiving ticagrelor as compared with $11.7 \%$ of those receiving clopidogrel $(P<0.001)$. This significantly lower event rate was driven by lower cardiovascular mortality, MI and stent thrombosis rates. The mortality benefit (4.5\% with ticagrelor vs. $5.9 \%$ with clopidogrel) contrasts with the TRITON-TIMI 38 trial, were no differences in mortality were observed. Mechanisms for the reduction in mortality potentially include the favourable balance between the atherothrombotic effect and bleeding risk due to the faster speed of action or the higher potency of platelet inhibition with ticagrelor, or mechanisms beyond pure P2Y12 receptor inhibition [37]. It might be directly related to the metabolism of adenosine. In addition to causing reversible platelet inhibition, adenosine is involved in numerous biological activities including cardioprotection from reperfusion injury, apoptosis, myocyte regeneration, improved myocardial contractility, and electrical stability. Another explanation might be the small difference in bleeding. Major bleeding, according to the PLATO study definition, occurred in $11.6 \%$ of the patients in the clopidogrel group versus $11.2 \%$ in the ticagrelor group $(2.2$ vs. 2.8 if the TIMI non-CABG-related major bleeding definition is used). In contrast to the use of prasugrel in TRITON-TIMI 38, there was no increased risk of CABG-related bleeding with ticagrelor. Comparable with prasugrel, non-procedure-related bleeding, including gastrointestinal and intracranial bleeding, were numerically higher with ticagrelor than with clopidogrel, although not statistically significant different. The prevention of ischemic events with ticagrelor is achieved by a greater antiplatelet effect in the first hours of treatment and during maintenance therapy [38]. Notably, ticagrelor was associated with dyspnea resulting discontinuation in $0.9 \%$ of the patients. Finally, ventricular pauses were observed more frequently in the ticagrelor group. In 3,000 patients with available continuous ECG monitoring, these were predominantly asymptomatic pauses, sinoatrial nodal in origin, and nocturnal that occurred most frequently in the acute phase of the index ACS. There were no clinical consequences related to the excess of these ventricular pauses in patients assigned to ticagrelor [39]. Subanalyses from the PLATO trial are summarized in Table 1.

\section{Cangrelor}

The comparison between cangrelor and clopidogrel have been described in the large phase III CHAMPION (Cangrelor versus Standard Therapy to Achieve Optimal Management of Platelet Inhibition) PCI and CHAMPION PLATFORM trials [40, 41]. The major difference between the two trials was the timing of the administration of the study drugs. In the CHAMPION PCI trial, cangrelor or clopidogrel $(600 \mathrm{mg})$ was started within $30 \mathrm{~min}$ before PCI. In the CHAMPION PLATFORM trial, cangrelor was started at the beginning of PCI, while clopidogrel $(600 \mathrm{mg})$ was administered at the end of PCI. In the 8,877 patients enrolled in CHAMPION PCI and 2655 enrolled in CHAMPION PLATFORM, no reduction in ischemic outcomes was observed at $48 \mathrm{~h}$ when comparing cangrelor with clopidogrel. In CHAMPION PLATFORM, cangrelor use was associated with reductions in the prespecified secondary outcomes stent thrombosis and death. Similar to the observation in PLATO, transient dyspnea occurred more often with cangrelor use. 


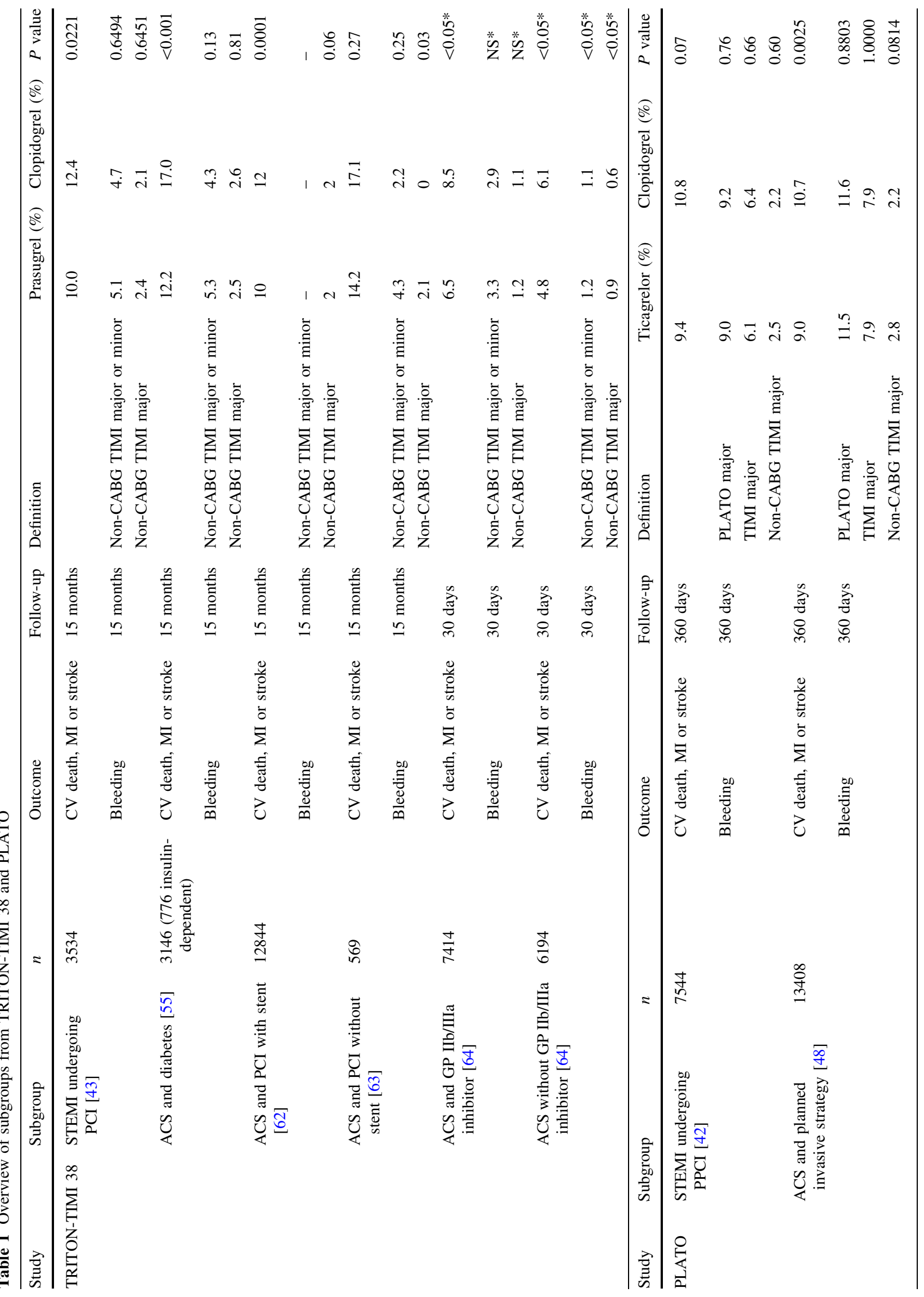




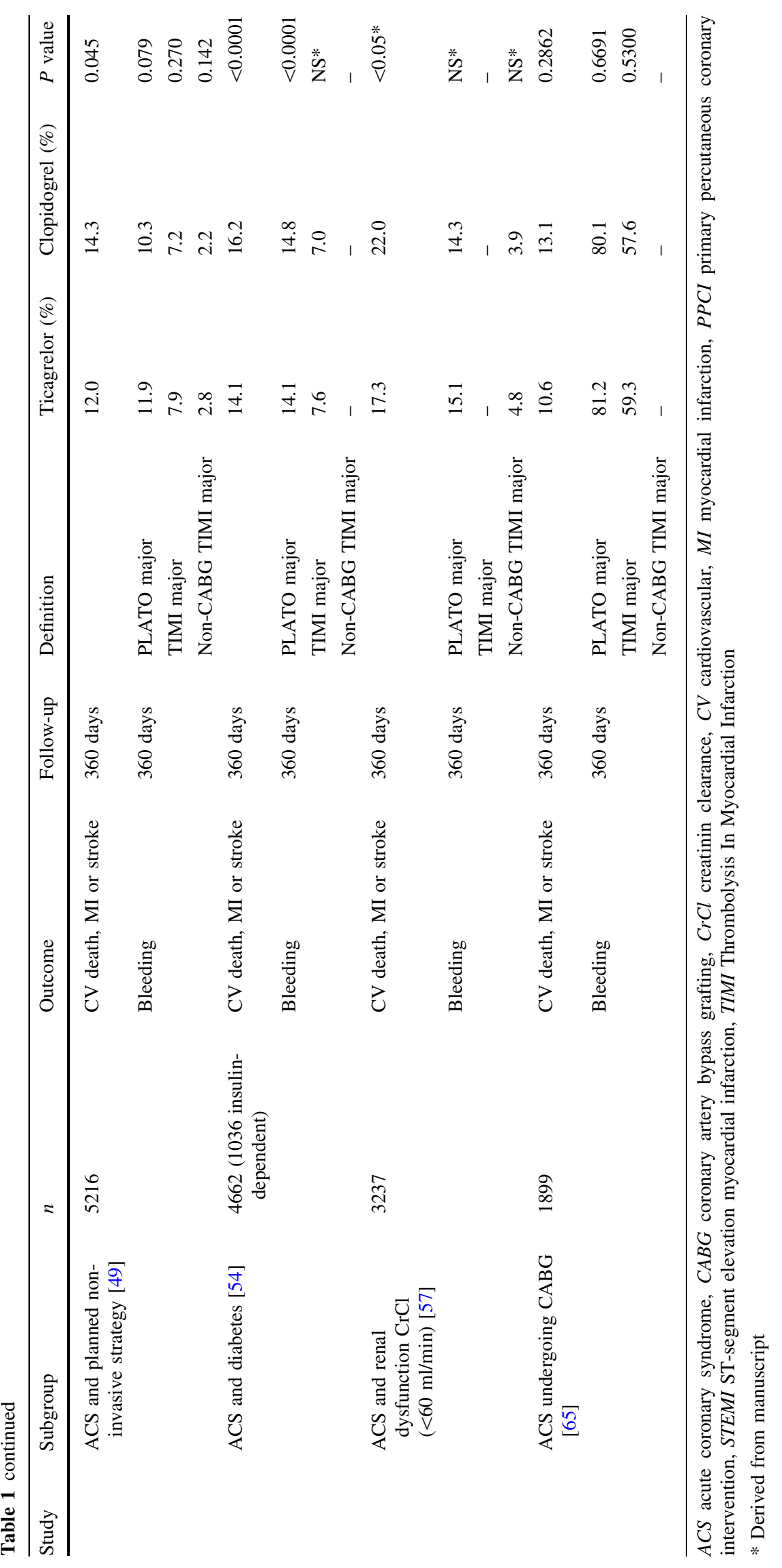




\section{Clinical practice}

The choice of drug, initiation, and duration of P2Y12 inhibition depend on the clinical setting (urgent or elective intervention) and patient-related factors such as the ischemic risk, bleeding risk and other baseline clinical characteristics. The levels of recommendation regarding P2Y12 inhibition are according to the European Society of Cardiology 2010 guidelines on myocardial revascularization.

\section{STEMI}

The preferred treatment for patients with STEMI is mechanical reperfusion by primary PCI. Thus fast acting P2Y12 inhibitors are of paramount importance in these high-risk patients who require urgent intervention. In STEMI patients, prasugrel $(60 \mathrm{mg}$ loading dose, followed by $10 \mathrm{mg}$ daily) or ticagrelor (180 mg loading dose, followed by $90 \mathrm{mg}$ twice daily) are recommended as P2Y12 inhibitors (both class I, level B). Both agents have been shown to reduce ischemic outcomes in STEMI patients, without increasing the bleeding risk significantly [42, 43]. Clopidogrel (600 mg loading dose, followed by $75 \mathrm{mg}$ daily) should be used primarily if the more effective ADP receptor blockers are contraindicated or unavailable (class I, level C).

\section{NSTE-ACS}

The choice of P2Y12 inhibition in patients presenting with NSTE-ACS depends on the chosen treatment strategy. Presently, there are three trials with long-term follow-up after a routine or selective invasive management in these patients [44-46]. Based on these clinical trial results and a large patient-pooled meta-analysis, current guidelines recommend a routine invasive strategy, consisting of routine coronary angiography and PCI if suitable, in high-risk NSTE-ACS patients [2, 47].

High risk of ischemic heart disease is associated with ST-segment changes, elevated troponin, diabetes, and a GRACE risk score of more than 140. ACS patients undergoing a routine invasive management have been analyzed in subgroup analyses from PLATO and TRITONTIMI 38. In PLATO, NSTE-ACS patients comprised around $50 \%$ of the ACS population, while around $75 \%$ were NSTE-ACS patients in TRITON-TIMI 38 [26, 48]. Compared to clopidogrel, ticagrelor or prasugrel reduced ischemic outcomes in these patients. For prasugrel and ticagrelor, there is respectively a class IIa, level B and class $\mathrm{I}$, level $\mathrm{B}$ recommendation. However, in patients with a history of stroke and TIA, or prasugrel is contraindicated and in patients with a body weight of less than $60 \mathrm{~kg}$, and patients aged $\geq 75$ years, prasugrel should be used with caution with a $5 \mathrm{mg}$ dose because of an increased bleeding risk. Prasugrel should also be avoided in patients referred for CABG. If prasugrel is used, it should be administered after coronary angiography.

Patients triaged to the selective invasive strategy undergo coronary angiography only in case of hemodynamic or electrical instability, or a positive ischemia detection test. With the latest trials focusing mainly on patients undergoing invasive management, less data is available on conservatively managed patients. However a substudy from PLATO in ACS patients intended for noninvasive management showed that the benefits of ticagrelor over clopidogrel are consistent, and with a greater absolute benefit with those from the overall PLATO results [49]. This indicates that ticagrelor can be recommended unless there are contra-indications to this agent.

Regardless of the intended treatment strategy in NSTEACS, a large proportion of the patients do not undergo revascularization during initial hospitalization. Because the optimal approach to antiplatelet therapy for high-risk, medically managed NSTE-ACS patients remains uncertain, the TaRgeted platelet Inhibition to cLarify the Optimal strateGy to medicallY manage Acute Coronary Syndromes (TRILOGY ACS) trial currently enrols medically managed NSTE-ACS patients who are randomized to prasugrel and aspirin versus clopidogrel and aspirin for a median duration of 18 months [50]. Regarding ticagrelor, the abovementioned PLATO substudy in ACS patients intended for a non-invasive management showed a consistent benefit of ticagrelor regardless of revascularization.

\section{Elective PCI}

After diagnostic coronary angiography, the majority of PCI procedures ultimately result in stent placement. The current European guidelines for myocardial revascularization recommend a clopidogrel loading dose of $300 \mathrm{mg}$ administered at least $6 \mathrm{~h}$ before the procedure (class I, level c) [2]. A $600 \mathrm{mg}$ loading dose may be preferred if given within 6 hours. If no intervention is planned after coronary angiography, clopidogrel can be stopped. In patients with a high thrombotic risk (diabetics, patients after recurrent MI, after stent thrombosis, complex lesions such as left main stenting, or in life threatening situations should an occlusion occur) or patients with a high on treatment platelet reactivity, clopidogrel might not optimally protect against thrombotic complications. However, the role of currently available platelet reactivity assays are unclear as the predictive accuracy of platelet function tests for ischemic outcomes is only modest [51]. This point was made clear in the aforementioned GRAVITAS trial, showing a higher clopidogrel dosing in patients with a high residual platelet activity did not reduce ischemic outcomes. In addition, the 
Testing Platelet Reactivity In Patients Undergoing Elective Stent Placement on Clopidogrel to Guide Alternative Therapy With Prasugrel (TRIGGER-PCI) trial (ClinicalTrials.gov identifier: NCT00910299) was recently terminated because of low event ( 6 month cardiovascular death or MI) rates. Although these trials question the use of routine platelet testing in elective PCI, it must first be mentioned that it has been shown that the predictive accuracy of platelet function tests for ischemic outcomes is only modest. Moreover, no test is able to identify patients at high risk for bleeding. Second, no data is available in the abovementioned patients at high risk for ischemic events, including comparisons between prasugrel and ticagrelor with clopidogrel. Third, although genotyping might assist in identifying patients with a low response to clopidogrel, CYP2C19 polymorphisms explain around $5.2 \%$ of the antiplatelet response [52]. No data is currently available regarding ticagrelor, prasugrel, or a high dose clopidogrel after platelet function testing in patients at high risk for ischemic events.

Other clinical considerations in the choice of P2Y12 inhibition

Diabetic patients, especially those with an ACS, are at a high risk for (recurrent) ischemic events. This can partly be attributed to increased platelet reactivity [53]. Substudies of PLATO and TRITON-TIMI 38 in diabetic patients confirmed this higher risk in diabetic patients. Ticagrelor reduced ischemic outcomes in ACS patients, irrespective of the diabetic status, when compared to clopidogrel but numerically more in patients with poor metabolic control (HbA1c >6\%) [54]. Total major bleeding events were similar, but non-CABG related major bleeding events were numerically more frequent with ticagrelor. When comparing prasugrel with clopidogrel in diabetic patients, a lower number of ischemic and similar bleeding events were observed [55]. Possible explanations for the similar bleeding rates remain speculative, but have been ascribe by investigators as possibly due to higher body weight or greater baseline platelet reactivity among diabetics, or simply the play of chance. The latter explanation is supported by the similar relative increase in the combination of major or minor bleeding among diabetics and nondiabetics and the higher major bleeding rate among diabetics compared with nondiabetics on clopidogrel. These findings were not expected if related to platelet reactivity only. Moreover, there was no significant interaction regarding the main outcome.

A high risk subgroup for bleeding events, often excluded from major clinical trials, are patients with an impaired renal function. The extent of renal dysfunction is related to cardiovascular outcomes, as illustrated in patients with renal dysfunction who presented with a MI [56]. Importantly, the initial dose of an antithrombotic drug does not add to the risk of bleeding in cases of renal dysfunction [2]. In contrast, repeated dosing results in accumulation of drugs and its associated increased bleeding risk. Dose reductions could potentially mitigate the accumulation of drugs. However, in patients with a glomerular filtration rate (GFR) of $30-60 \mathrm{ml} / \mathrm{min} / 1.73 \mathrm{~m}^{2}$, no information is available about dose reductions for prasugrel. Moreover, prasugrel is contraindicated in patients with severe renal dysfunction (GFR of less than $30 \mathrm{ml} / \mathrm{min} / 1.73 \mathrm{~m}^{2}$ ). Regarding clopidogrel, no information is available about dose reductions in patients with renal dysfunction. Finally, for ticagrelor no reduction is required in patients with a GFR of less than $60 \mathrm{ml} / \mathrm{min} / 1.73 \mathrm{~m}^{2}$ and the benefit was sustained and in fact numerically enhanced in this high risk subset of patients [57].

Ticagrelor is dosed twice daily which requires good adherence to medical therapy to ensure a reduction in risk of ischemic events, although this might also be true for the other medication required once daily. Treatment with any of the more potent P2Y12 inhibitors results in higher risk for spontaneous bleeding events, accumulating over time which should be considered in frail patients.

In patients with ACS, in which an invasive management is planned, the presence of shock, vomiting or sedation might prevent oral intake of P2Y12 inhibitors. In these patients, intravenous cangrelor might prove to be an alternative for the oral agents. However, cangrelor is currently not approved for clinical practice.

\section{Conclusions and remaining questions}

New P2Y12 inhibitors have decreased ischemic events after PCI compared with clopidogrel [58]. On the horizon is elinogrel, a novel P2Y12 inhibitor that has passed the dose-escalation study ERASE MI (Early Rapid Reversal of Platelet Thrombosis with Intravenous Elinogrel before PCI to Optimize Reperfusion in Acute Myocardial Infarction) [59]. Results of ERASE MI were incorporated into the design of the phase II nonurgent INNOVATE PCI trial (INtraveNous and Oral administration of elinogrel, a selective and reversible P2Y [12]-receptor inhibitor, versus clopidogrel to eVAluate Tolerability and Efficacy in nonurgent Percutaneous Coronary Interventions; ClinicalTrials.gov identifier: NCT00751231) were both IV and oral dosing of elinogrel are compared with clopidogrel (presented by Rao SV et al., at European Society of Cardiology Conference 2010). Patients treated with elinogrel, a reversible and competitive P2Y12-receptor antagonist which requires no metabolic activation, had greater inhibition of platelet aggregation than those treated with 
clopidogrel. At 120 days, there was no difference in major bleeding, minor bleeding, or bleeding requiring medical attention among those treated with elinogrel and those treated with clopidogrel. Currently, a phase III trial is planned.

Despite the improvement in clinical outcomes with the new P2Y12 inhibitors, remaining questions are the possibility to switch drugs, different doses, duration of therapy, optimal time of initiation (in NSTE-ACS), and costeffectiveness.

Regarding the duration of therapy, current clinical practice guidelines recommend 12-month treatment with dual antiplatelet therapy in the setting of ACS. However, the optimal duration after PCI and the extent to which dual antiplatelet therapy confers benefit against ischemic events (including stent thrombosis) beyond 12 months is not known. The DAPT study is currently enrolling patients to assess the impact of 30 versus 12 months of dual antiplatelet therapy in patients undergoing PCI with stent placement [60].

Cost-effectiveness of the novel agents ticagrelor and prasugrel have been recently described. From the US healthcare perspective, treatment with prasugrel versus clopidogrel for a median of 14.7 months appeared to be an economically dominant treatment strategy, resulting in both lower costs and greater life expectancy [61]. The lower costs were mainly due to a reduction in the costs of repeat PCIs. A cost effectiveness study of ticagrelor from PLATO showed that treating ACS patients with ticagrelor for 12 months is associated with a gain in 0.13 qualityadjusted life year in a lifetime perspective compared with generic clopidogrel (presented by Henriksson $\mathrm{M}$ et al. at the International Society for Pharmacoeconomics and Outcomes Research Conference 2011). Regarding the cost per quality-adjusted life year gained, ticagrelor was highly cost-effective applying conventional thresholds of costeffectiveness.

Disclosures Stefan K James declares research grants and advisory board fees from AstraZeneca and honorariums from AstraZeneca, Bristol-Myers Squibb, Schering-Plough, Merck, and Eli-Lilly. All other authors have no disclosures.

Open Access This article is distributed under the terms of the Creative Commons Attribution Noncommercial License which permits any noncommercial use, distribution, and reproduction in any medium, provided the original author(s) and source are credited.

\section{References}

1. Jennings LK (2009) Mechanisms of platelet activation: need for new strategies to protect against platelet-mediated atherothrombosis. Thromb Haemost 102(2):248-257
2. Wijns W, Kolh P, Danchin N, et al (2010) Guidelines on myocardial revascularization: The Task Force on Myocardial Revascularization of the European Society of Cardiology (ESC) and the European Association for Cardio-Thoracic Surgery (EACTS). Eur Heart J Sep 25

3. Silber S, Albertsson P, Avilés FF et al (2005) Guidelines for percutaneous coronary interventions. Eur Heart J 26(8):804-847

4. Meadows TA, Bhatt DL (2007) Clinical aspects of platelet inhibitors and thrombus formation. Circ Res 100(9):1261-1275

5. Mehran R, Rao SV, Bhatt DL et al (2011) Standardized bleeding definitions for cardiovascular clinical trials: a consensus report from the bleeding academic research consortium. Circulation 123(23):2736-2747

6. Chen ZM, Jiang LX, Chen YP et al (2005) Addition of clopidogrel to aspirin in 45,852 patients with acute myocardial infarction: randomised placebo-controlled trial. Lancet 366(9497):16071621

7. Sabatine MS, Cannon CP, Gibson CM et al (2005) Addition of clopidogrel to aspirin and fibrinolytic therapy for myocardial infarction with ST-segment elevation. N Engl J Med 352(12): 1179-1189

8. Yusuf S, Zhao F, Mehta SR et al (2001) Effects of clopidogrel in addition to aspirin in patients with acute coronary syndromes without ST-segment elevation. N Engl J Med 345(7):494-502

9. Davi G, Patrono C (2007) Platelet activation and atherothrombosis. N Engl J Med 357(24):2482-2494

10. Dorsam RT, Kunapuli SP (2004) Central role of the P2Y12 receptor in platelet activation. J Clin Invest 113(3):340-345

11. Wallentin L (2009) P2Y(12) inhibitors: differences in properties and mechanisms of action and potential consequences for clinical use. Eur Heart J 30(16):1964-1977

12. Ben-Dor I, Kleiman NS, Lev E (2009) Assessment, mechanisms, and clinical implication of variability in platelet response to aspirin and clopidogrel therapy. Am J Cardiol 104(2):227-233

13. Bates ER, Lau WC, Angiolillo DJ (2011) Clopidogrel-drug interactions. J Am Coll Cardiol 57(11):1251-1263

14. Buonamici P, Marcucci R, Migliorini A et al (2007) Impact of platelet reactivity after clopidogrel administration on drug-eluting stent thrombosis. J Am Coll Cardiol 49(24):2312-2317

15. Gurbel PA, Bliden KP, Samara W et al (2005) Clopidogrel effect on platelet reactivity in patients with stent thrombosis: results of the CREST Study. J Am Coll Cardiol 46(10):1827-1832

16. Hochholzer W, Trenk D, Bestehorn HP et al (2006) Impact of the degree of peri-interventional platelet inhibition after loading with clopidogrel on early clinical outcome of elective coronary stent placement. J Am Coll Cardiol 48(9):1742-1750

17. Wenaweser P, Dorffler-Melly J, Imboden K et al (2005) Stent thrombosis is associated with an impaired response to antiplatelet therapy. J Am Coll Cardiol 45(11):1748-1752

18. Farid NA, Kurihara A, Wrighton SA (2010) Metabolism and disposition of the thienopyridine antiplatelet drugs ticlopidine, clopidogrel, and prasugrel in humans. J Clin Pharmacol 50(2): 126-142

19. Mega JL, Close SL, Wiviott SD et al (2009) Cytochrome p-450 polymorphisms and response to clopidogrel. N Engl J Med 360(4):354-362

20. Wallentin L, James S, Storey RF et al (2010) Effect of CYP2C19 and $\mathrm{ABCB} 1$ single nucleotide polymorphisms on outcomes of treatment with ticagrelor versus clopidogrel for acute coronary syndromes: a genetic substudy of the PLATO trial. Lancet 376(9749):1320-1328

21. Shuldiner AR, O'Connell JR, Bliden KP et al (2009) Association of cytochrome P450 2C19 genotype with the antiplatelet effect and clinical efficacy of clopidogrel therapy. JAMA 302(8): 849-857 
22. Price MJ, Berger PB, Teirstein PS et al (2011) Standard- vs highdose clopidogrel based on platelet function testing after percutaneous coronary intervention: the GRAVITAS randomized trial. JAMA 305(11):1097-1105

23. Jakubowski JA, Winters KJ, Naganuma H, Wallentin L (2007) Prasugrel: a novel thienopyridine antiplatelet agent. A review of preclinical and clinical studies and the mechanistic basis for its distinct antiplatelet profile. Cardiovasc Drug Rev 25(4):357-374

24. Scott DM, Norwood RM, Parra D (2009) P2Y12 inhibitors in cardiovascular disease: focus on prasugrel. Ann Pharmacother 43(1):64-76

25. Jernberg T, Payne CD, Winters KJ et al (2006) Prasugrel achieves greater inhibition of platelet aggregation and a lower rate of nonresponders compared with clopidogrel in aspirin-treated patients with stable coronary artery disease. Eur Heart J 27(10): 1166-1173

26. Wiviott SD, Trenk D, Frelinger AL et al (2007) Prasugrel compared with high loading- and maintenance-dose clopidogrel in patients with planned percutaneous coronary intervention: the prasugrel in comparison to clopidogrel for inhibition of platelet activation and aggregation-thrombolysis in myocardial infarction 44 trial. Circulation 116(25):2923-2932

27. Husted S, Emanuelsson H, Heptinstall S et al (2006) Pharmacodynamics, pharmacokinetics, and safety of the oral reversible P2Y12 antagonist AZD6140 with aspirin in patients with atherosclerosis: a double-blind comparison to clopidogrel with aspirin. Eur Heart J 27(9):1038-1047

28. Storey RF, Husted S, Harrington RA et al (2007) Inhibition of platelet aggregation by AZD6140, a reversible oral P2Y12 receptor antagonist, compared with clopidogrel in patients with acute coronary syndromes. J Am Coll Cardiol 50(19):1852-1856

29. Storey RF, Oldroyd KG, Wilcox RG (2001) Open multicentre study of the P2T receptor antagonist AR-C69931MX assessing safety, tolerability and activity in patients with acute coronary syndromes. Thromb Haemost 85(3):401-407

30. Storey RF, Wilcox RG, Heptinstall S (2002) Comparison of the pharmacodynamic effects of the platelet ADP receptor antagonists clopidogrel and AR-C69931MX in patients with ischaemic heart disease. Platelets 13(7):407-413

31. Wiviott SD, Antman EM, Winters KJ et al (2005) Randomized comparison of prasugrel (CS-747, LY640315), a novel thienopyridine P2Y12 antagonist, with clopidogrel in percutaneous coronary intervention: results of the Joint Utilization of Medications to Block Platelets Optimally (JUMBO)-TIMI 26 trial. Circulation 111(25):3366-3373

32. Wiviott SD, Braunwald E, McCabe $\mathrm{CH}$ et al (2007) Prasugrel versus clopidogrel in patients with acute coronary syndromes. N Engl J Med 357(20):2001-2015

33. Bhatt DL (2007) Intensifying platelet inhibition-navigating between Scylla and Charybdis. N Engl J Med 357(20):2078-2081

34. Pocock SJ, Mehran R, Clayton TC et al (2010) Prognostic modeling of individual patient risk and mortality impact of ischemic and hemorrhagic complications: assessment from the Acute Catheterization and Urgent Intervention Triage Strategy Trial. Circulation 121(1):43-51

35. Cannon CP, Husted S, Harrington RA et al (2007) Safety, tolerability, and initial efficacy of AZD6140, the first reversible oral adenosine diphosphate receptor antagonist, compared with clopidogrel, in patients with non-ST-segment elevation acute coronary syndrome: primary results of the DISPERSE-2 trial. J Am Coll Cardiol 50(19):1844-1851

36. Wallentin L, Becker RC, Budaj A et al (2009) Ticagrelor versus clopidogrel in patients with acute coronary syndromes. N Engl J Med 361(11):1045-1057

37. Serebruany VL, Atar D (2010) The PLATO trial: do you believe in magic? Eur Heart J 31(7):764-767
38. Storey RF, Angiolillo DJ, Patil SB et al (2010) Inhibitory effects of ticagrelor compared with clopidogrel on platelet function in patients with acute coronary syndromes: the PLATO (PLATelet inhibition and patient Outcomes) PLATELET substudy. J Am Coll Cardiol 56(18):1456-1462

39. Scirica BM, Cannon CP, Emanuelsson $\mathrm{H}$ et al (2011) The incidence of bradyarrhythmias and clinical bradyarrhythmic events in patients with acute coronary syndromes treated with ticagrelor or clopidogrel in the PLATO (Platelet Inhibition and Patient Outcomes) Trial Results of the Continuous Electrocardiographic Assessment Substudy. J Am Coll Cardiol 57(19):1908-1916

40. Harrington RA, Stone GW, McNulty S et al (2009) Platelet inhibition with cangrelor in patients undergoing PCI. N Engl J Med 361(24):2318-2329

41. Bhatt DL, Lincoff AM, Gibson CM et al (2009) Intravenous platelet blockade with cangrelor during PCI. N Engl J Med 361(24):2330-2341

42. Steg PG, James S, Harrington RA et al (2010) Ticagrelor versus clopidogrel in patients with ST-elevation acute coronary syndromes intended for reperfusion with primary percutaneous coronary intervention: a Platelet Inhibition and Patient Outcomes (PLATO) trial subgroup analysis. Circulation 122(21):21312141

43. Montalescot G, Wiviott SD, Braunwald E et al (2009) Prasugrel compared with clopidogrel in patients undergoing percutaneous coronary intervention for ST-elevation myocardial infarction (TRITON-TIMI 38): double-blind, randomised controlled trial. Lancet 373(9665):723-731

44. Fox KA, Poole-Wilson P, Clayton TC et al (2005) 5-Year outcome of an interventional strategy in non-ST-elevation acute coronary syndrome: the British Heart Foundation RITA 3 randomised trial. Lancet 366(9489):914-920

45. Lagerqvist B, Husted S, Kontny F et al (2006) 5-Year outcomes in the FRISC-II randomised trial of an invasive versus a noninvasive strategy in non-ST-elevation acute coronary syndrome: a follow-up study. Lancet 368(9540):998-1004

46. Damman P, Hirsch A, Windhausen F, Tijssen JG, de Winter RJ (2010) 5-Year clinical outcomes in the ICTUS (Invasive versus Conservative Treatment in Unstable coronary Syndromes) trial a randomized comparison of an early invasive versus selective invasive management in patients with non-ST-segment elevation acute coronary syndrome. J Am Coll Cardiol 55(9):858-864

47. Fox KA, Clayton TC, Damman P et al (2010) Long-term outcome of a routine versus selective invasive strategy in patients with non-ST-segment elevation acute coronary syndrome a metaanalysis of individual patient data. J Am Coll Cardiol 55(22): $2435-2445$

48. Cannon CP, Harrington RA, James S et al (2010) Comparison of ticagrelor with clopidogrel in patients with a planned invasive strategy for acute coronary syndromes (PLATO): a randomised double-blind study. Lancet 375(9711):283-293

49. James SK, Roe MT, Cannon CP et al (2011) Ticagrelor versus clopidogrel in patients with acute coronary syndromes intended for non-invasive management: substudy from prospective randomised PLATelet inhibition and patient Outcomes (PLATO) trial. BMJ 342:d3527

50. Chin CT, Roe MT, Fox KA et al (2010) Study design and rationale of a comparison of prasugrel and clopidogrel in medically managed patients with unstable angina/non-ST-segment elevation myocardial infarction: the TaRgeted platelet Inhibition to cLarify the Optimal strateGy to medicallY manage Acute Coronary Syndromes (TRILOGY ACS) Trial. Am Heart J 160(1):16-22

51. Breet NJ, van Werkum JW, Bouman HJ et al (2010) Comparison of platelet function tests in predicting clinical outcome in patients undergoing coronary stent implantation. JAMA 303(8):754-762 
52. Hochholzer W, Trenk D, Fromm MF et al (2010) Impact of cytochrome P450 2C19 loss-of-function polymorphism and of major demographic characteristics on residual platelet function after loading and maintenance treatment with clopidogrel in patients undergoing elective coronary stent placement. J Am Coll Cardiol 55(22):2427-2434

53. Angiolillo DJ, Fernandez-Ortiz A, Bernardo E et al (2005) Platelet function profiles in patients with type 2 diabetes and coronary artery disease on combined aspirin and clopidogrel treatment. Diabetes 54(8):2430-2435

54. James S, Angiolillo DJ, Cornel JH et al (2010) Ticagrelor vs. clopidogrel in patients with acute coronary syndromes and diabetes: a substudy from the PLATelet inhibition and patient Outcomes (PLATO) trial. Eur Heart J 31(24):3006-3016

55. Wiviott SD, Braunwald E, Angiolillo DJ et al (2008) Greater clinical benefit of more intensive oral antiplatelet therapy with prasugrel in patients with diabetes mellitus in the trial to assess improvement in therapeutic outcomes by optimizing platelet inhibition with prasugrel-thrombolysis in myocardial infarction 38. Circulation 118(16):1626-1636

56. Anavekar NS, McMurray JJ, Velazquez EJ et al (2004) Relation between renal dysfunction and cardiovascular outcomes after myocardial infarction. N Engl J Med 351(13):1285-1295

57. James S, Budaj A, Aylward P et al (2010) Ticagrelor versus clopidogrel in acute coronary syndromes in relation to renal function: results from the Platelet Inhibition and Patient Outcomes (PLATO) Trial. Circulation 122(11):1056-1067

58. Bellemain-Appaix A, Brieger D, Beygui F et al (2010) New P2Y12 inhibitors versus clopidogrel in percutaneous coronary intervention: a meta-analysis. J Am Coll Cardiol 56(19): $1542-1551$

59. Berger JS, Roe MT, Gibson CM et al (2009) Safety and feasibility of adjunctive antiplatelet therapy with intravenous elinogrel, a direct-acting and reversible P2Y12 ADP-receptor antagonist, before primary percutaneous intervention in patients with ST-elevation myocardial infarction: the Early Rapid ReversAl of platelet thromboSis with intravenous Elinogrel before PCI to optimize reperfusion in acute Myocardial Infarction (ERASE MI) pilot trial. Am Heart J 158(6):998-1004

60. Mauri L, Kereiakes DJ, Normand SL et al (2010) Rationale and design of the dual antiplatelet therapy study, a prospective, multicenter, randomized, double-blind trial to assess the effectiveness and safety of 12 versus 30 months of dual antiplatelet therapy in subjects undergoing percutaneous coronary intervention with either drug-eluting stent or bare metal stent placement for the treatment of coronary artery lesions. Am Heart J 160(6):1035-1041 1041.e.1

61. Mahoney EM, Wang K, Arnold SV et al (2010) Cost-effectiveness of prasugrel versus clopidogrel in patients with acute coronary syndromes and planned percutaneous coronary intervention: results from the trial to assess improvement in therapeutic outcomes by optimizing platelet inhibition with Prasugrel-Thrombolysis in Myocardial Infarction TRITON-TIMI 38. Circulation 121(1):71-79

62. Wiviott SD, Braunwald E, McCabe CH et al (2008) Intensive oral antiplatelet therapy for reduction of ischaemic events including stent thrombosis in patients with acute coronary syndromes treated with percutaneous coronary intervention and stenting in the TRITON-TIMI 38 trial: a subanalysis of a randomised trial. Lancet 371(9621):1353-1363

63. Pride YB, Wiviott SD, Buros JL et al (2009) Effect of prasugrel versus clopidogrel on outcomes among patients with acute coronary syndrome undergoing percutaneous coronary intervention without stent implantation: a TRial to assess Improvement in Therapeutic Outcomes by optimizing platelet inhibitioN with prasugrel (TRITON)-Thrombolysis in Myocardial Infarction (TIMI) 38 substudy. Am Heart J 158(3):e21-e26

64. O'Donoghue M, Antman EM, Braunwald E et al (2009) The efficacy and safety of prasugrel with and without a glycoprotein IIb/IIIa inhibitor in patients with acute coronary syndromes undergoing percutaneous intervention: a TRITON-TIMI 38 (Trial to Assess Improvement in Therapeutic Outcomes by Optimizing Platelet Inhibition With Prasugrel-Thrombolysis In Myocardial Infarction 38) analysis. J Am Coll Cardiol 54(8):678-685

65. Held C, Asenblad N, Bassand JP et al (2011) Ticagrelor versus clopidogrel in patients with acute coronary syndromes undergoing coronary artery bypass surgery: results from the PLATO (Platelet Inhibition and Patient Outcomes) trial. J Am Coll Cardiol 57(6):672-684 